\title{
Nucleated red blood cells and soluble transferrin receptor in thalassemia syndromes: relationship with global and ineffective erythropoiesis
}

\author{
Paolo Danise ${ }^{1, *}$, Giovanni Amendola ${ }^{2}$, Rosanna \\ Di Concilio², Enrico Cillari ${ }^{3}$, Maria Gioia ${ }^{3}$, Anna \\ Di Palma ${ }^{1}$, Daniela Avino ${ }^{1}$, Paolo Rigano ${ }^{4}$ and \\ Aurelio Maggio 4 \\ ${ }^{1}$ Department of Laboratory of Hematology, \\ “Umberto 1" Hospital, Nocera Inferiore, Salerno, \\ Italy \\ ${ }^{2}$ Department of Pediatrics and Neonatal Intensive \\ Care Unit - Pediatric Hematology and Oncology

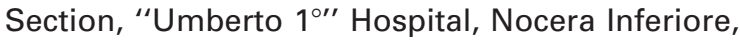 \\ Salerno, Italy \\ ${ }^{3}$ Department of Clinical Pathology, "V. Cervello" \\ Hospital, Palermo, Italy \\ ${ }^{4}$ Second Hematology Unit, "V. Cervello" Hospital, \\ Palermo, Italy
}

\begin{abstract}
Background: The technology to recognize nucleated red blood cells (NRBC) automatically has only recently been developed. Modern hematology analyzers allow for rapid and accurate NRBC counts. The goal of our study was to evaluate NRBC counts and the concentrations of serum transferrin receptor (sTfR) in patients affected by different thalassemia syndromes and hereditary spherocytosis. We wished to gain a better understanding of the meaning of the presence of NRBC in peripheral blood and the relationship of the two parameters with effective and ineffective erythropoiesis in the different thalassemia syndromes.

Methods: NRBC counts in peripheral blood were evaluated in a large group of patients with thalassemia (36 thalassemia major, 55 thalassemia intermedia and $61 \mathrm{~S} \beta$-thalassemia patients) and compared with data from 29 patients with hereditary spherocytosis; in all the patients the concentration of sTfR was evaluated as an index of global erythropoiesis.

Results: The NRBC count showed a good relationship with ineffective erythropoiesis: highest counts were observed in the thalassemia syndromes characterized by almost completely ineffective erythropoiesis. NRBCs were absent in patients affected by hereditary spherocitosis, a disease characterized by effective erythropoiesis.

Conclusions: The NRBC count can be useful for better defining ineffective erythropoiesis in patients with

\footnotetext{
*Corresponding author: Paolo Danise, MD, Department of Laboratory of Hematology, "Umberto $1^{\circ \prime}$ " Hospital, Via S. Francesco 1, Nocera Inferiore (Sa), 84014, Italy Phone: +390819213470, Fax: +390819213370,

E-mail: p.danise@libero.it

Received July 3, 2009; accepted August 28, 2009; previously published online October 13, 2009
}

thalassemia, and can help optimize transfusion therapy in severe thalassemia syndromes.

Clin Chem Lab Med 2009;47:1539-42.

Keywords: erythropoiesis; hereditary spherocytosis; nucleated red blood cells; serum transferrin receptor; thalassemia syndromes.

\section{Introduction}

The presence of nucleated red blood cells (NRBC) in the peripheral blood of patients with thalassemia syndromes has long been known (1). Unfortunately, it is difficult to obtain fast and accurate counts of NRBC using traditional microscopy (2). The technology to recognize such cells automatically has only recently been developed, and modern hematology analyzers have made fast and accurate NRBC counts possible $(3,4)$. In the past, hematology analyzers only gave a flag specific to NRBCs (5) and it was difficult to utilize the NRBC count as a useful hematological parameter. In 1969, it was suggested that a NRBC count of $<5 \%$ in transfusion-dependent thalassemia patients was a good indication that the patient was being treated appropriately $(6,7)$. Subsequently, it has been shown that the severity of erythropoietic stress, as measured by the NRBC count, was suppressed when the hemoglobin concentration was maintained above $10 \mathrm{~g} / \mathrm{dL}$ (8), thus indicating that the pool of NRBCs in circulation was a reflection of the degree of ineffective erythropoiesis in thalassemia syndromes (9). Previous studies showed that the concentration of serum transferrin receptors (sTfR) was the most reliable parameter for the evaluation of global erythropoiesis, either effective or ineffective (10-12). The goal of the present study was the evaluation of NRBC counts and the concentration of STfR in a large group of patients affected by different thalassemia syndromes and hereditary spherocytosis. We wished to gain a better understanding of the meaning of the presence of NRBCs in the peripheral blood of patients, and the relationship of the two parameters with effective and ineffective erythropoiesis in the different thalassemia syndromes.

\section{Materials and methods}

\section{Study population}

The study population included all patients with severe thalassemia syndromes and hereditary spherocytosis regularly followed in the Authors' Departments. This included 36 
patients with thalassemia major ( 17 males, 29 females, mean age $=21$ years, range 3-31 years, 20 splenectomized), 55 patients with thalassemia intermedia ( 25 males, 30 females, mean age $=33$ years, range $18-63$ years, 32 splenectomized), 61 patients with $S \beta$-thalassemia ( 35 males, 26 females, mean age $=41$ years, range 18-63 years, 31 splenectomized) and 29 patients with hereditary spherocytosis (13 males, 16 females, mean age $=16$ years, range $5-44$ years, 9 splenectomized). The diagnosis was based on current clinical and laboratory criteria and patients received extensive molecular analysis. The study was approved by the Ethical Committee of "Umberto 1" Hospital, Nocera Inferiore (SA), and informed consent was obtained from all patients or their guardians in accordance with institutional guidelines. Samples were collected prior to blood transfusion in patients on chronic transfusion therapy, and at least 3 months following transfusion in all other patients.

\section{NRBC count}

The Sysmex XE-2100 analyzer (Sysmex Corporation, Kobe, Japan) gives an accurate count of NRBCs with very good correlation to both traditional microscopy and flow cytometry, the reference method (5). A specific reagent (fluorochrome polymethine dye) completely lyses red blood cells and, at the same time, enucleates, shrinks and slightly stains the NRBC nuclei. The lysing reagent maintains the shape of the white blood cells and intensively stains their intra-cytoplasmatic organelles and nuclei. Differences in the staining of NRBC nuclei and white blood cells, as well as their different volumes, are detected by a semiconductor laser using forward-scattered light and fluorescence. This allows for clear separation of the two cell populations. The values of NRBCs are expressed as the absolute number $\times 10^{9} / \mathrm{L}$.

\section{sTfR assay}

The assay was performed using an automated immunoturbidimetric assay method (IEMA) that utilized a monoclonal antibody (Idea s-TfR-It, Orion diagnostica, Espoo, Finland), and measured with an LX 800 analyzer (Beckman Coulter, Brea, CA, USA). The concentrations of sTfR were expressed as $\mathrm{mg} / \mathrm{L}$ and compared with those measured in 50 normal controls $(1.23 \pm 0.23 \mathrm{mg} / \mathrm{L}, 25$ males, 25 females, mean age $=32$ years, range $10-49$ years).

\section{Statistics}

The median value of each parameter in the splenectomized and non-splenectomized groups was evaluated using the $p$ value of the Mann-Whitney test to assess if the distribution of a single parameter in the two groups was statistically different $(p<0.001)$. The distribution of $s T f R$ and NRBC values was non-Gaussian. Therefore, non-parametric statistical tests such as Spearman's correlation was used. This test assesses the degree of correlation ( $r$-values) between parameter pairs. Positive r-values indicate an indirect correlation (if one parameter increases, the other decreases): parameters were considered to be significantly correlated if they had a p-value $<0.01$.

\section{Results}

NRBCs were absent in patients with hereditary spherocytosis, and present in 36/36 patients with thalassemia major (100\% of cases). NRBCs were also present in $48 / 55$ patients with thalassemia intermedia

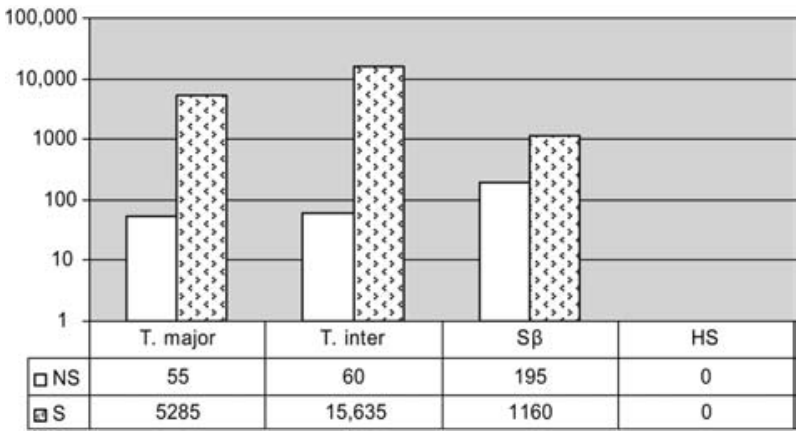

Figure 1 Median values of NRBC counts in non-splenectomized patients and in splenectomized patients.

NRBC, nucleated red blood cell (values are expressed as absolute number $\times 10^{9} / \mathrm{L}$ ). T. major, thalassemia major; $\mathrm{T}$. inter, thalassemia intermedia; $\mathrm{S} \beta, \mathrm{S} \beta$-thalassemia; HS, hereditary spherocytosis; NS, non-splenectomized patients; $\mathrm{S}$, splenectomized patients.

patients (87\% of cases) and in $48 / 61$ patients with $\mathrm{S} \beta$ thalassemia ( $79 \%$ of cases). Figures 1 and 2 show the median values of the NRBC counts and STfR concentrations in non-splenectomized and splenectomized patients, respectively. As expected, NRBC counts were always higher in splenectomized patients, with the highest counts in patients with thalassemia intermedia. In non-splenectomized patients, the highest counts were found in patients with $S \beta$-thalassemia. Patients with thalassemia intermedia showed the highest concentrations of sTfR.

Table 1 shows the median values and statistical difference in NRBCs and sTfR concentrations in splenectomized and non-splenectomized patients with thalassemia major, intermedia, $\mathrm{S} \beta$-thalassemia and hereditary spherocytosis. The NRBC counts showed a significant difference only between non-splenectomized and splenectomized patients in the different thalassemia syndromes. The absence of a spleen does not allow for removal of erythroblasts. As a consequence, it makes measurement of circulating NRBCs a more reliable index of ineffective erythropoiesis in these patients. Table 2 shows the correlation between median values of the NRBC count and STfR concen-

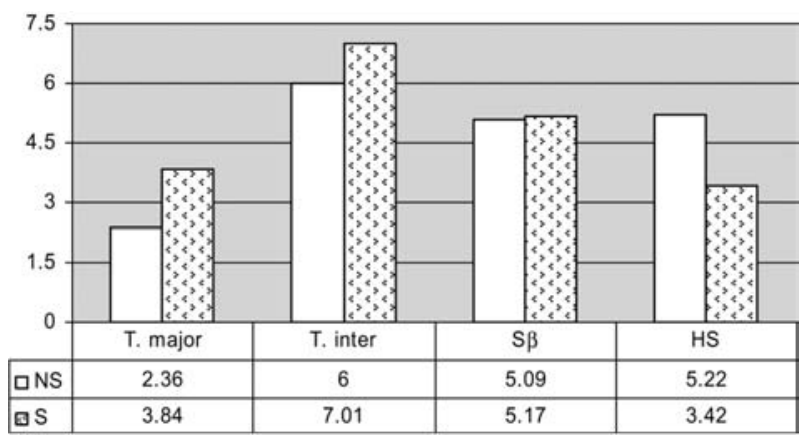

Figure 2 Median values of sTfR levels in non-splenectomized patients and in splenectomized patients.

sTfR, serum transferrin receptor (levels are expressed as $\mathrm{mg} / \mathrm{L})$. T. major, thalassemia major; T. inter, thalassemia intermedia; $\mathrm{S} \beta, \mathrm{S} \beta$-thalassemia; $\mathrm{HS}$, hereditary spherocytosis; NS, non-splenectomized patients; $S$, splenectomized patients. 
Table 1 Median values and statistical differences in non-splenectomized and splenectomized patients.

\begin{tabular}{|c|c|c|c|c|c|c|c|c|c|c|c|c|}
\hline & \multicolumn{3}{|c|}{ T. major } & \multicolumn{3}{|c|}{ T. intermedia } & \multicolumn{3}{|c|}{$\mathrm{S} \beta$-thalassemia } & \multicolumn{3}{|c|}{ Hereditary spherocytosis } \\
\hline & No sp & Splen & p-Value & No $s p$ & Splen & $p$-Value & No sp & Splen & p-Value & No $s p$ & Splen & p-Value \\
\hline $\begin{array}{l}\text { NRBC \# } \\
\text { sTfR }\end{array}$ & $\begin{array}{l}55 \\
2.36\end{array}$ & $\begin{array}{l}5.285 \\
3.84\end{array}$ & $\begin{array}{l}<0.0001 \\
0.3556 \text { NS }\end{array}$ & $\begin{array}{l}60 \\
6.00\end{array}$ & $\begin{array}{c}15.635 \\
7.01\end{array}$ & $\begin{array}{l}<0.0001 \\
0.1749 \mathrm{NS}\end{array}$ & $\begin{array}{l}195 \\
5.09\end{array}$ & $\begin{array}{l}1.190 \\
5.17\end{array}$ & $\begin{array}{l}0.0004 \\
0.6652 \text { NS }\end{array}$ & $\begin{array}{l}0 \\
5.22\end{array}$ & $\begin{array}{l}0 \\
3.42\end{array}$ & $\begin{array}{l}- \\
<0.0009\end{array}$ \\
\hline
\end{tabular}

T. major, thalassemia major; T. intermedia, thalassemia intermedia; no sp, non-splenectomized; splen, splenectomized; NRBC, nucleated red blood cell; sTfR, serum transferrin receptor.

trations in patients with thalassemia. The NRBC count and STfR concentrations showed statistically significant correlation in splenectomized patients only.

\section{Discussion}

During the last few decades, technology has provided us with very sophisticated hematology analyzers and new hematological parameters that are often introduced before their full clinical potential is realized. Recently, a rapid, automated and accurate NRBC count has become available, allowing the determination of this parameter in very large groups of patients with thalassemia (3). The analysis of data from this study shows that NRBCs were present in the blood of all patients with thalassemia major, and all but seven patients with thalassemia intermedia; these seven patients had extremely mild clinical features and a genotype characterized by co-inheritance of severe $\beta_{+}$ or $\beta_{\text {o }}$ alleles with triplicated $\alpha$-globin genes $(\alpha \alpha \alpha / \alpha \alpha$ or $\alpha \alpha \alpha / \alpha \alpha \alpha)$. NRBCs were particularly increased in splenectomized subjects, as previously reported (3-8). NRBC counts in non-splenectomized patients with $\mathrm{S} \beta$-thalassemia were higher than in non-splenectomized patients with thalassemia major and intermedia. It is possible that progressive functional asplenia, which occurs over time in patients with $\mathrm{S} \beta$ thalassemia, makes them more similar to splenectomized patients and thus increasing the NRBC count. Splenectomized patients with thalassemia intermedia, who have the most prominent clinical features of ineffective erythropoiesis, showed the highest NRBC counts. The lowest counts were found in patients with $S \beta$-thalassemia who have much lower ineffective erythropoiesis. These data suggest that automated NRBC counts could be a useful parameter of the

Table 2 Degree of correlation between NRBC counts and sTfR in patients with thalassemia.

\begin{tabular}{lll}
\hline & \multicolumn{2}{l}{ NRBC \# vs. sTfR } \\
\cline { 2 - 3 } & No $\mathrm{sp}$ & Splen \\
\hline T. major & $\mathrm{r} 0.54$ & $\mathrm{r} 0.64$ \\
$\mathrm{~T}$ T. intermedia & $\mathrm{p} 0.1059$ & $\mathrm{p} 0.0008$ \\
$\mathrm{r} 0.24$ & $\mathrm{r} 0.58$ \\
S $\beta$-thalassemia & $\mathrm{p} 0.2637$ & $\mathrm{p} 0.0005$ \\
& $\mathrm{r}-0.31$ & $\mathrm{r} 0.51$ \\
& $\mathrm{p} 0.0913$ & $\mathrm{p} 0.0033$ \\
\hline
\end{tabular}

NRBC, nucleated red blood cell; sTfR, serum transferrin receptor; no sp, non-splenectomized; splen, splenectomized; T. major, thalassemia major; T. intermedia, thalassemia intermedia. degree of ineffective erythropoiesis $(10,11)$. The concentrations of sTfR in thalassemia patients and patients with hereditary spherocytosis were increased, while NRBCs were present only in the peripheral blood of patients with thalassemia. sTfR is a reliable estimate of the erythroid compartment mass, together with erythron transferrin uptake (ETU), a ferrokinetic measurement which is not used routinely since it involves the administration of radioactive iron (10-14). sTfR concentrations are directly proportional to the number of transferrin receptors on erythroblasts, which in normal subjects falls within a narrow range (15). However, sTfR concentrations do not distinguish between effective and ineffective erythropoiesis. STfR concentrations are similar in patients with hereditary spherocytosis and severe thalassemia syndromes, as clearly shown by our data (Figure 2). The analysis of combined data from NRBC counts and sTfR concentrations showed that patients with thalassemia major had moderately elevated NRBC counts and moderately high concentrations of sTfR. In these patients global erythropoiesis is only mildly increased due to suppression by transfusion therapy, but residual erythropoiesis is ineffective. Patients with thalassemia intermedia, which is characterized by a high degree of global ineffective erythropoiesis (7), had high concentrations of STfR and high NRBC counts. Non-splenectomized patients with $\mathrm{S} \beta$-thalassemia showed increased NRBC counts, while splenectomized patients with $\mathrm{S} \beta$-thalassemia had fewer NRBCs than splenectomized patients with thalassemia major and intermedia. However, sTfR concentrations were increased in both groups of patients with $\mathrm{S} \beta$-thalassemia, according to high global and low ineffective erythropoiesis in these patients. Patients with hereditary spherocytosis showed an absence of circulating NBRCs and high sTfR concentrations, in accordance with their increased but completely effective erythropoiesis.

In conclusion, the automated determination of NRBC counts could be useful in the evaluation of patients affected by thalassemia syndromes. This parameter could provide further information not currently deducible from other parameters. The different patterns of NRBC counts and sTfR concentrations suggest that the combined use of both parameters in patients with thalassemia could allow physicians to better define global erythropoiesis and the degree of ineffective erythropoiesis in these patients. This will allow transfusion therapy in patients with severe thalassemia syndromes to be given with greater accuracy. 


\section{Conflict of interest statement}

All the Authors declare that they have not accepted any funding or support from an organization that may in any way gain or lose financially from the results of their study or the conclusions of their review, they have not been employed by an organization that may in any way gain or lose financially from the results of their study or the conclusions of their review, and do not have any other conflicting interests.

\section{References}

1. Clifford GO. The clinical significance of leukoerythroblastic anemia. Med Clin North Am 1966;50:779-90.

2. Li J. The preliminary study of nucleated red blood cell counting by automated haematology analyzer. Sysmex J Int 2004;14:13-7.

3. Paterakis G, Kossivas L, Kendall R, Anagnostopoulos N, Stavropoulos-Giokas, Tassiopoulou A. Comparative evaluation of the erythroblast count generated by three-color fluorescence flow cytometry, the Abbott Cell-Dyn 4000 hematology analyzer, and microscopy. Lab Hematol 1998;4:64-70.

4. Gulati G, Behling E, Kocher W, Schwarting R. An evaluation of the performance of Sysmex XE-2100 in enumerating nucleated red cells in peripheral blood. Arch Pathol Lab Med 2007;131:1077-83.

5. Briggs C, Harrison P, Grant D, Staves J, MacHin SJ. New quantitative parameters on a recently introduced automated blood cell counter - the XE 2100. Clin Lab Haematol 2000;22:345-50.

6. Piomelli S, Danoff SJ, Becker MH, Lipera MJ, Travis SF. Prevention of bone malformations and cardiomegaly in
Cooley's anemia by early hypertransfusion regimen. Ann NY Acad Sci 1969;165:427-36.

7. Fosburg MT, Nathan DG. Treatment of Cooley's anemia. Blood 1990;76:435-44.

8. Rachmilewitz EA, Thorell B. Characterization of the normoblast population in beta-thalassemia blood by rapidflow cytofluorimetry. Scand J Haematol 1978;20:440-6.

9. Sher G, Viitisallo B, Schisano T, Pantalony D, Van Hove L. Automated NRBC count, a new parameter to monitor, in real time, individualized transfusion needs in transfusion-dependent thalassemia major. Lab Hematol 1997; 3:129-37.

10. Beguin Y, Clemons GK, Pootrakul P, Fillet G. Quantitative assessment of erythropoiesis and functional classification of anemia based on measurement of serum transferrin receptor and erythropoietin. Blood 1993;81:106776.

11. Galanello R, Barella S, Turco MP, Giagu N, Cao A, Dore $F$, et al. Serum erythropoietin and erythropoiesis in high and low-fetal hemoglobin beta-thalassemia intermedia patients. Blood 1994;83:561-5.

12. Cazzola M, De Stefano P, Ponchio L, Locatelli F, Beguin $Y$, Dessi C, et al. Relationship between transfusion regimen and suppression of erythropoiesis in betathalassemia major. Br J Haematol 1995;89:473-8.

13. Cazzola M, Pootrakul P, Huebers HA, Eng M, Eschbach $\mathrm{J}$, Finch CA. Erythroid marrow function in anemic patients. Blood 1997;69:296-301.

14. Huebers HA, Beguin Y, Pootrakul P, Einspahr D, Finch CA. Intact transferrin receptors in human plasma and their relation to erythropoiesis. Blood 1990;75:102-7.

15. Kuiper-Kramer PA, Huisman CM, Van der Molen-Sinke J, Abbes A, Van Eijk HG. The expression of transferrin receptors on erythroblasts in anaemia of chronic disease, myelodysplastic syndromes and iron deficiency. Acta Haematol 1997;97:127-31. 